\title{
Relationship between amino acid properties and functional parameters in olfactory receptors and discrimination of mutants with enhanced specificity
}

\author{
M Michael Gromiha ${ }^{1 *}$, K Harini $^{2}$, R Sowdhamini ${ }^{2}$, Kazuhiko Fukui ${ }^{3}$ \\ From The 2011 International Conference on Intelligent Computing (ICIC 2011) \\ Zhengzhou, China. 11-14 August 2011
}

\begin{abstract}
Background: Olfactory receptors are key components in signal transduction. Mutations in olfactory receptors alter the odor response, which is a fundamental response of organisms to their immediate environment. Understanding the relationship between odorant response and mutations in olfactory receptors is an important problem in bioinformatics and computational biology. In this work, we have systematically analyzed the relationship between various physical, chemical, energetic and conformational properties of amino acid residues, and the change of odor response/compound's potency/half maximal effective concentration (EC50) due to amino acid substitutions.

Results: We observed that both the characteristics of odorant molecule (ligand) and amino acid properties are important for odor response and EC50. Additional information on neighboring and surrounding residues of the mutants enhanced the correlation between amino acid properties and EC50. Further, amino acid properties have been combined systematically using multiple regression techniques and we obtained a correlation of 0.90-0.98 with odor response/EC50 of goldfish, mouse and human olfactory receptors. In addition, we have utilized machine learning methods to discriminate the mutants, which enhance or reduce EC50 values upon mutation and we obtained an accuracy of $93 \%$ and $79 \%$ for self-consistency and jack-knife tests, respectively.
\end{abstract}

Conclusions: Our analysis provides deep insights for understanding the odor response of olfactory receptor mutants and the present method could be used for identifying the mutants with enhanced specificity.

\section{Background}

Membrane proteins perform several functions, including the transport of ions and molecules across the membrane, binding to small molecules at the extracellular space, recognizing the immune system and energy transducers. Olfactory receptors (OR) are membrane proteins, belonging to the G Protein-Coupled Receptor superfamily, which are characterized by the presence of hydrophobic transmembrane domains. The odorant response of an organism by ORs to its environment forms the basis for our understanding in intra-species interactions, hostpathogen interactions, balance of chemicals, cell-cell

\footnotetext{
* Correspondence: gromiha@iitm.ac.in

'Department of Biotechnology, Indian Institute of Technology Madras, Chennai 600 036, Tamilnadu, India

Full list of author information is available at the end of the article
}

interactions and other fundamental processes. It is evident that individual odorant can be recognized by multiple ORs and conversely, one type of OR can recognize multiple odorants with distinct binding affinities and specificities [1,2]. The binding and response of ORs with odorants are critical for the conversion of chemical information into electronic signals in olfactory sensory neurons $[3,4]$. Recent studies showed that mosquitoes' odorant receptors help the insects to find humans and, inadvertently, to transmit malaria $[5,6]$. Further, ORs have been analyzed to understand the mechanism of chloride uptake [7], modulation of signaling [8], functional architecture of olfactory system [9], unitary response [10], structural and functional plasticity at binding pocket [11] etc. Similar analysis has also been reported for identifying

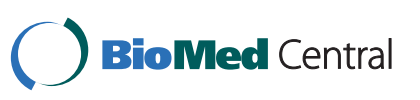

(c) 2012 Gromiha et al.; licensee BioMed Central Ltd. This is an Open Access article distributed under the terms of the Creative Commons Attribution License (http://creativecommons.org/licenses/by/2.0), which permits unrestricted use, distribution, and reproduction in any medium, provided the original work is properly cited. 
the binding site residues and binding specificity of proteinprotein complexes [12-17].

The importance of specific amino acid residues in ORs and other membrane proteins has been demonstrated through site-directed mutagenesis experiments. The experimental data on EC50, maximal velocity of transport, odorant response, percentage uptake of compounds, affinity and specificity have been accumulated in the database for functional residues in membrane proteins [18]. Kuang et al. [19] measured the EC50 values for lysine in the wild type and mutants of 5.24 receptor. Luu et al. [20] elucidated the features of olfactory receptors for determining ligand specificity using different amino acid agonists. The structural basis for mouse OR to EC50 data has been analyzed by systematically substituting amino acid residues in different transmembrane helical segments [2,21]. Schmiedeberg et al. [22] carried out docking studies to understand the influence of different chemical compounds as well as due to mutations. On the other hand, computational methods have been proposed to understand the binding affinity of ligands with ORs using the template structure of rhodopsin [23-25].

In spite of these studies, the role of amino acid properties for the change of EC50 or odorant response has not yet been explored. Further, it is necessary to develop computational models to discriminate the mutants, which increase or decrease EC50. In this work, we have constructed different datasets of goldfish, mouse and human ORs for the mutants that change the odorant response, increase cAMP (adenosine 3'-5'-cyclic mono phosphate) and EC50 values. The differences in experimental data (EC50/odor response etc.) upon mutations have been related with physical, chemical, energetic and conformational properties of amino acid residues and the important amino acid properties have been brought out. The combinations of amino acid properties and the influence of neighboring and surrounding residues have been successfully used to relate the experimental functional data. Further, machine learning methods have been utilized to discriminate mutants with enhanced EC50 values.

\section{Materials and methods Datasets}

We have developed a database, TMFunction, for functionally important amino acid residues in membrane proteins [18]. TMFunction has been searched for all functional data available for ORs. We obtained the experimental data, EC50, odorant response and cAMP increase for goldfish, mouse and human ORs. The final dataset contains 119 data with the following categories: (i) EC50: goldfish OR with Lys, 12; Arg : 12; Gly: 6 and
Glu: 6; mouse OR: 28; Human OR: 7; (ii) odorant response: cAMP increase: 24 and $\mathrm{Ca}^{2+}$ increase: 24 .

\section{Amino acid properties}

We used a set of 49 diverse amino acid properties (physical, chemical, energetic and conformational), which fall into various clusters analyzed by Tommi and Kanehisa [26] in the present study. The amino acid properties were normalized between 0 and 1 using the expression:

$$
P_{\text {norm }}(i)=\left[P(i)-P_{\min }\right] /\left[P_{\max }-P_{\min }\right],
$$

where $\mathrm{P}(\mathrm{i}), \mathrm{P}_{\text {norm }}(\mathrm{i})$ are, respectively, the original and normalized values of amino acid i for a particular property, and $\mathrm{P}_{\min }$ and $\mathrm{P}_{\max }$ are, respectively, the minimum and maximum values. Further, the numerical and normalized values for all the 49 properties used in this study along with their brief descriptions have been explained in our earlier articles $[27,28]$ and are available on the web at http://www.cbrc.jp/ gromiha/fold_rate/ property.html. These properties have been successfully used to understand the folding and stability of proteins [29-33].

\section{Computational procedure}

The mutation induced changes in property values $\Delta \mathrm{P}(\mathrm{i})$ was computed using the equation [29]:

$$
\Delta \mathrm{P}(\mathrm{i})=\mathrm{P}_{\text {mut }}(\mathrm{i})-\mathrm{P}_{\text {wild }}(\mathrm{i})
$$

where $\mathrm{P}_{\text {mut }}(\mathrm{i})-\mathrm{P}_{\text {wild }}(\mathrm{i})$ are, respectively, the property value of the ith mutant and wild type residues, and $i$ varies from 1 to $\mathrm{N}$; total number of mutants. The computed difference in property values $\Delta \mathrm{P}$ ( $\mathrm{X}$ in Eqn. 3) was related with experimental EC50 or odorant response ( $\mathrm{Y}$ in Eqn. 3) using single correlation coefficient (r). It is given by:

$$
\mathrm{r}=\left[N \sum_{i=1}^{N} X_{i} Y_{i}-\left(\sum_{i=1}^{N} X_{i} \sum_{i=1}^{N} Y_{i}\right)\right] / \sqrt{\left[N \sum_{i=1}^{N} X_{i}^{2}-\left(\sum_{i=1}^{N} X_{i}\right)^{2}\right]\left[N \sum_{i=1}^{N} Y_{i}^{2}-\left(\sum_{i=1}^{N} Y_{i}\right)^{2}\right]},
$$

\section{Molecular modelling of mouse OR73}

Mouse olfactory receptor 73 (OR73) sequence was obtained from NCBI (http://www.ncbi.nlm.nih.gov/ guide/) using text search. The TM regions and topology (N-terminus OUT/IN) of the sequence were predicted using the transmembrane prediction server HMMTOP [34]. The sequences of OR73 and the template (bovine rhodopsin; PDB ID: 1F88A) were aligned using PRALINE-TM [35] server and manually edited using JALVIEW [36] Version 2.4. The TM region predictions from HMMTOP and the two conserved motifs MAYDRYVAIC and NPXXY in OR73 were used to guide and improve the alignment of the query and the template. 
The crystal structure of the bovine rhodopsin [23] (PDB ID: 1f88 chain A) was used as a template for the comparative modelling of the query (OR73). The structure of the template was obtained from RCSB (http:// www.rcsb.org/pdb). The coordinates corresponding to the residues 236-239 and 328-333 were not available in the crystal structure of 1F88 chain A due to poor electron density and hence these residues were removed from the template sequence before the alignment. The final alignment (Figure 1) was used to construct the model using the software MODELLER [37] (version 9.8). A set of 20 structures were generated from which the five least probability density function models were validated by using PROCHECK [38] (Ramachandran Plot). The best structure was further energy minimized using the SYBYL software package (version 7.2) (Tripos Associates Inc.). Tripos force field, using 100 iterations of Powell's gradient with a distance dependent dielectric constant of 1 and a non bonded interaction cut off value of 8 and was terminated at a convergence of $0.05 \mathrm{kcal} \mathrm{mol}$. The final structures were further validated using PROCHECK [38].
Local sequence and structural effects

The effect of local sequence, $P_{\text {seq }}(i)$, was included using the equation [29]:

$$
p_{\text {seq }}(\mathrm{i})=\left[\sum_{j=i-k}^{j=i+k} P_{\mathrm{j}}(\mathrm{i})\right]-P_{\text {mut }}(\mathrm{i})
$$

where, $P_{\text {mut }}(\mathrm{i})$ is the property value of the ith mutant residue and $\Sigma P_{\mathrm{j}}(\mathrm{i})$ is the total property value of the segment of $(2 k+1)$ residues ranging from $i-k$ to $i+k$ about the $\mathrm{i}^{\text {th }}$ residue of wild type.

The structural information, $P_{\text {str }}(\mathrm{i})$, was included using the equation:

$$
P_{\text {str }}(\mathrm{i})=P_{\text {sur }}(\mathrm{i})-P_{\text {mut }}(\mathrm{i})
$$

where $P_{\text {mut }}(\mathrm{i})$ is the property value of the $\mathrm{i}^{\text {th }}$ mutant residue, and:

$$
P_{\text {sur }}(\mathrm{i})=\sum_{j} \mathrm{n}_{\mathrm{ij}} \cdot P_{\mathrm{j}}
$$

where, $\mathrm{n}_{\mathrm{ij}}$ is the total number of type $\mathrm{j}$ residues surrounding the $\mathrm{i}^{\text {th }}$ residue of the protein within the

\section{F88 YVPFSNKTGVVRSPFEAPQYYLAEPWQFSMLAAYMFLLIMLGFPINFLTL Or73 MTLSDGNHSGAVFTLLGFSDYPEL---TIPLFLIFLTIYSITVVGNIGMI TM1 \\ 1F88 YVTVQHKKLRTPLNYILLNLAVADLFMVFGGFTTTLYTSLHGYFVFGPT Or73 VIIRINPKLHIPMYFFLSHLSFVDFCYSSIVAPKMLVNLVTMNRI $\begin{array}{ll}\text { 1F88 } & \text { CNLEGFFATLGGEIALWSLVVLAIERYVVVCKPMSNFRFGENH-AIMGVA } \\ \text { Or73 CLVQFFFFCTFVVTESFLLGVMAYDRFVAIRNPLLYTVAMSQRLCAMLVL }\end{array}$ 1F88 FTWVMALACAAPPLVG - - - WSRYIPEGMQCSCGIDYYTPHEE - - - TNNE Or73 GSYAWGVVCSLILTCSALNLSFYGFNMINHFFCEFSSLLSLSRSDTSVSQ TM4 \\ 1F88 SFVIYMFVHFI IPL IVIFFCYGQLVFTVKEAAASATTQKAEKEVTRMVI Or73 LLLFVFATFNEISTLLIILLSYVLIVVTILKMKSASGRRKAFSTCASHLT \\ 1F88 IMVIAFLICWLPYAGVAFYIFTHQFMTIPAFFAKTSAVYNPVIYIMMNKQ Or73 AITIFHGTILFLYCVPNSKNSRHTVKVASVFYTVVIPMLNPLIYSLRNKD 1F88 FRNCMVTTLCCGKNPS* pr73 VKDTVKKIIGTKVYSS *}

Figure 1 Alignment of query (OR73) with template (1F88). Observed and predicted TM helix positions are marked in VIGBYOR representation. Key functional motifs are shown in pink color against OR73 sequence. 
volume of $8 \AA$, and $P_{\mathrm{j}}$ is the property value of residue type $j$. Further details about the computation of surrounding residues have been described in our earlier articles $[39,40]$.

\section{Multiple regression analysis}

We have combined the amino acid properties using multiple regression technique: multiple correlation coefficients and regression equations were determined using standard procedures [41]. When fitting the data by multiple regression technique, reducing the number of variables increases the reliability of results. Hence, we selected three to five properties by searching all possible combinations of the 49 properties and computed the multiple correlation coefficients for all data sets. The highest correlation coefficient was selected and used in the analysis.

\section{Machine learning methods}

We have analyzed several machine learning techniques implemented in WEKA program [42] for discriminating mutants with enhanced EC50 values. This program includes several methods based on Bayes function, Neural network, Radial basis function network, Logistic function, Support vector machine, Regression analysis, Nearest neighbor, Meta learning, Decision tree and Rules. The details of all these methods are available in our earlier articles [43-45].

\section{Jack-knife test}

We have performed a jack-knife (leave-one-out crossvalidation) test for assessing the validity of the present work. In this method, n-1 data are used for training and the left out mutant is used for testing.

\section{Assessment of predictive ability}

We have used different measures, such as sensitivity, specificity and accuracy to assess the performance of discriminating mutants with enhanced function. These terms are defined as follows:

$$
\begin{aligned}
& \text { Sensitivity }=\mathrm{TP} /(\mathrm{TP}+\mathrm{FN}) \\
& \text { Specificity }=\mathrm{TN} /(\mathrm{TN}+\mathrm{FP}) \\
& \text { Accuracy }=(\mathrm{TP}+\mathrm{TN}) /(\mathrm{TP}+\mathrm{TN}+\mathrm{FP}+\mathrm{FN}),
\end{aligned}
$$

where, TP, FP, TN and FN refer to the number of true positives, false positives, true negatives and false negatives, respectively.

\section{Results and discussion}

Relationship between amino acid properties and change in EC50 upon mutation: goldfish OR with Lys potency

We have computed the changes in amino acid properties using Eqn.2 and related them with changes in EC50 upon mutations using Eqn. 3. The accessible surface area of unfolding showed a negative correlation of -0.86 for Lys potency with goldfish OR, and other physical properties, bulkiness, volume etc. showed appreciable negative correlation with $\triangle \mathrm{EC} 50$ [46]. Figure 2a shows the relationship between $\triangle \mathrm{ASA}$ and $\triangle \mathrm{EC} 50$. On the

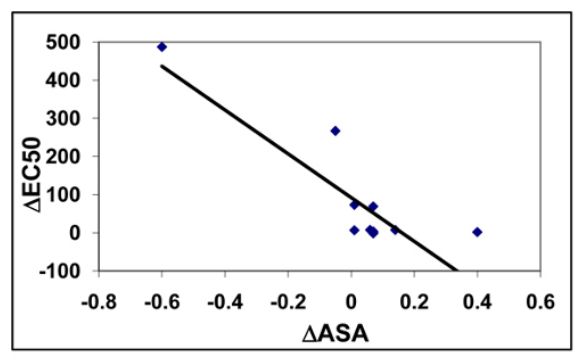

Figure 2a

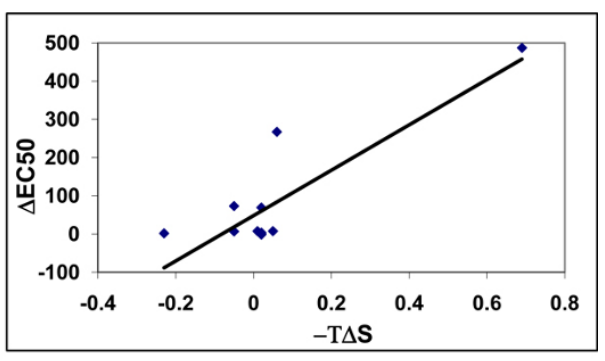

Figure 2b

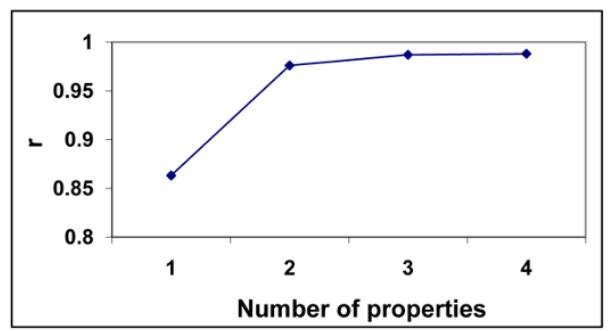

Figure 2c

Figure 2 (a) Relationship between $\triangle A S A$ and $\triangle E C 50$. (b) Variation of $-T \triangle S$ with $\triangle E C 50$. (c) Variation of correlation coefficient with number of properties. 
other hand, entropy change has high positive correlation with $\triangle \mathrm{EC} 50$. Figure $2 \mathrm{~b}$ shows the trend between - $\mathrm{T} \Delta \mathrm{S}$ and $\triangle \mathrm{EC} 50$ and the correlation coefficient is 0.85 .

We have analyzed the combined effect of different amino acid properties and related with $\triangle \mathrm{EC} 50$ values. The variation of correlation coefficient with number of properties is shown in Figure 2c. We noticed that the combination of four properties raised the correlation up to 0.988 .

\section{Arg potency}

The data on Arg potency with goldfish OR showed a maximum correlation of 0.62 with the property, reduction in accessibility. Further, the combination of five properties also showed the maximum correlation of 0.90 . Hence, we have included the information of neighboring residues along the sequence using Eqn. 4. We used different window lengths and the results for window lengths 3 , 5 and 7 for the combination of 2,3, and 4 amino acid properties is shown in Figure 3a.

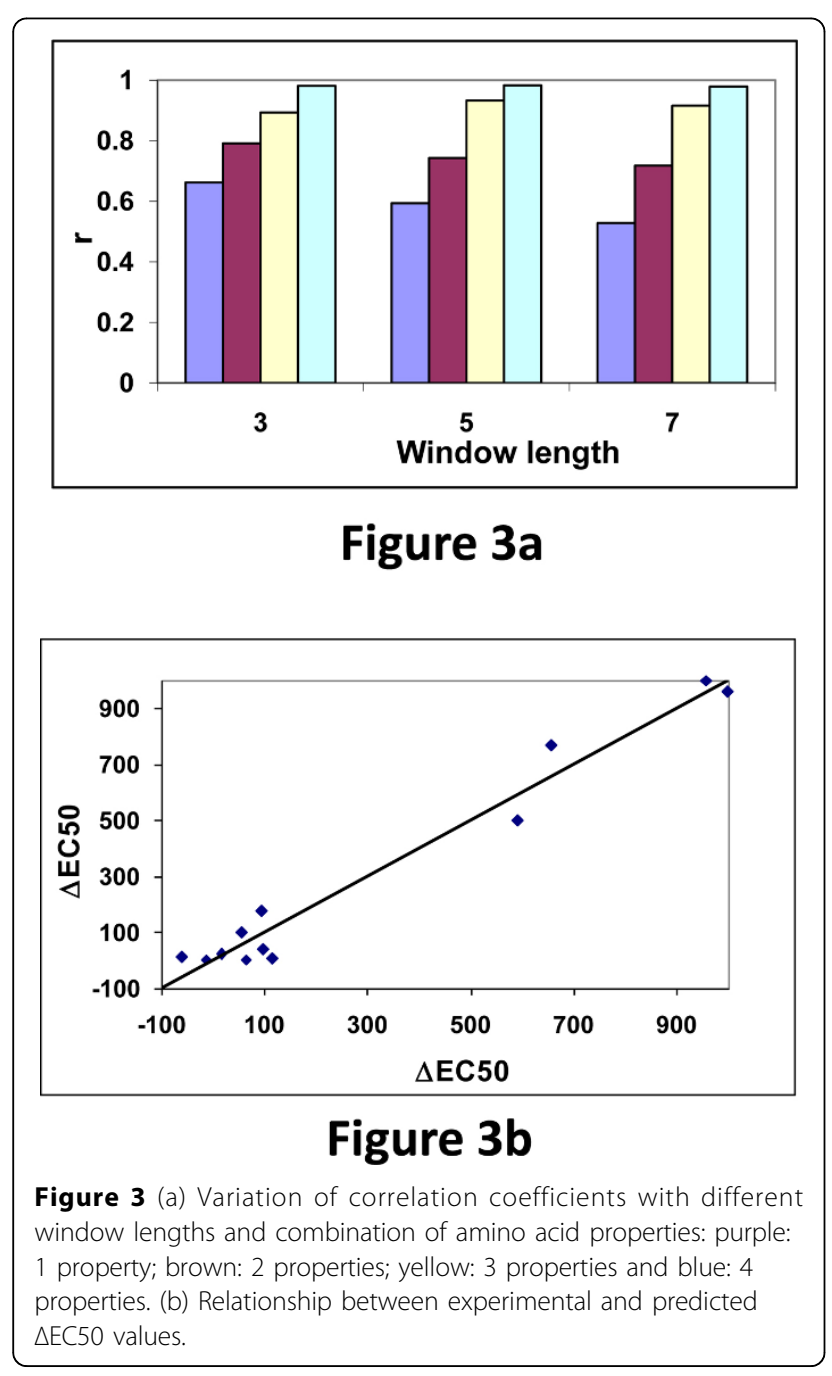

We observed that the combination of four properties raised the correlation up to 0.98 . This result shows that the experimental EC50 values are not depending on a specific residue and the information on neighboring residues are very important for the variation of EC50 upon mutation. The experimental and observed $\triangle \mathrm{EC} 50$ values for all the 12 mutants are shown in Figure $3 \mathrm{~b}$ and we noticed a good relationship between them. Further, the thermodynamic properties, $\Delta \mathrm{G}, \Delta \mathrm{H}$ and $-\mathrm{T} \Delta \mathrm{S}$ showed good correlation with $\triangle \mathrm{EC} 50$ for goldfish OR with Glu and Gly potency.

Molecular modeling and structural analysis of mouse OR A three-dimensional model of the mouse olfactory receptor, OR73, was obtained as mentioned in Methods. PROCHECK results for the model, excluding the loop regions, show more than $95 \%$ of residues in allowed regions (including strictly allowed and partially allowed) of the Ramachandran plot. The full-length structures of the models show more than $90 \%$ in the allowed regions

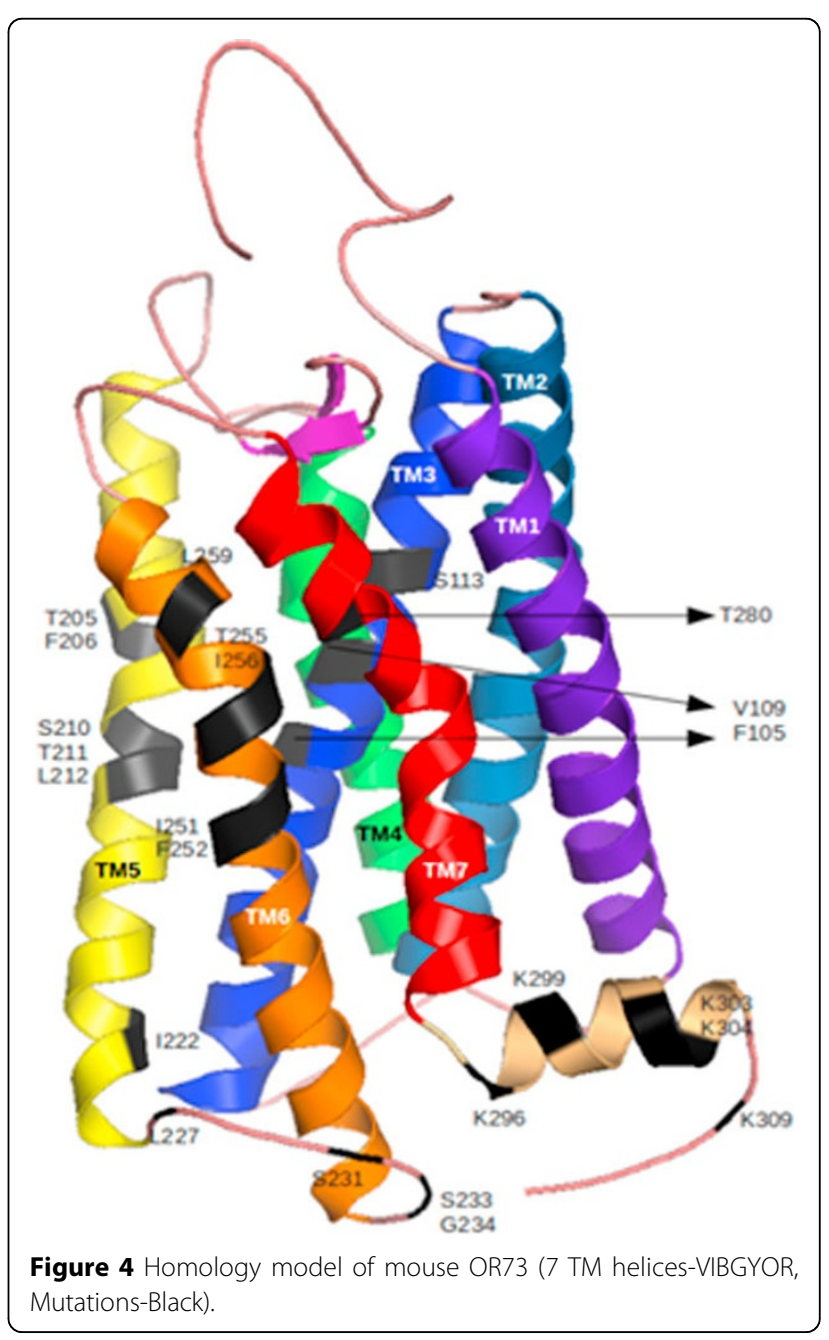


(including strictly allowed and partially allowed) of the Ramachandran plot. The residues that were found in the disallowed regions were mainly in the loop regions which are highly variable in length and sequence identity. The RMSD of the full length OR model with respect to template was found to be less than $2 \AA$.The amino acid mutations under study were marked on the model as shown in Figure 4. Many of the mutants lie in TM5 and TM6 and the loop in between has a glycine residue and this region could be implicated in the dimer interface. Most of the other 24 mutants are in intracellular loops.

The solvent accessibility of the amino acid residues, chosen for this study, were observed in the three-dimensional model. These values were calculated using PSA [47] within JOY version 3.2 [48,49] (Additional file 1). It is interesting that only 7 out of 24 mutants are solventburied and three of them are in TM3. Such solvent-buried residues could be important for the structural integrity of a protomer.

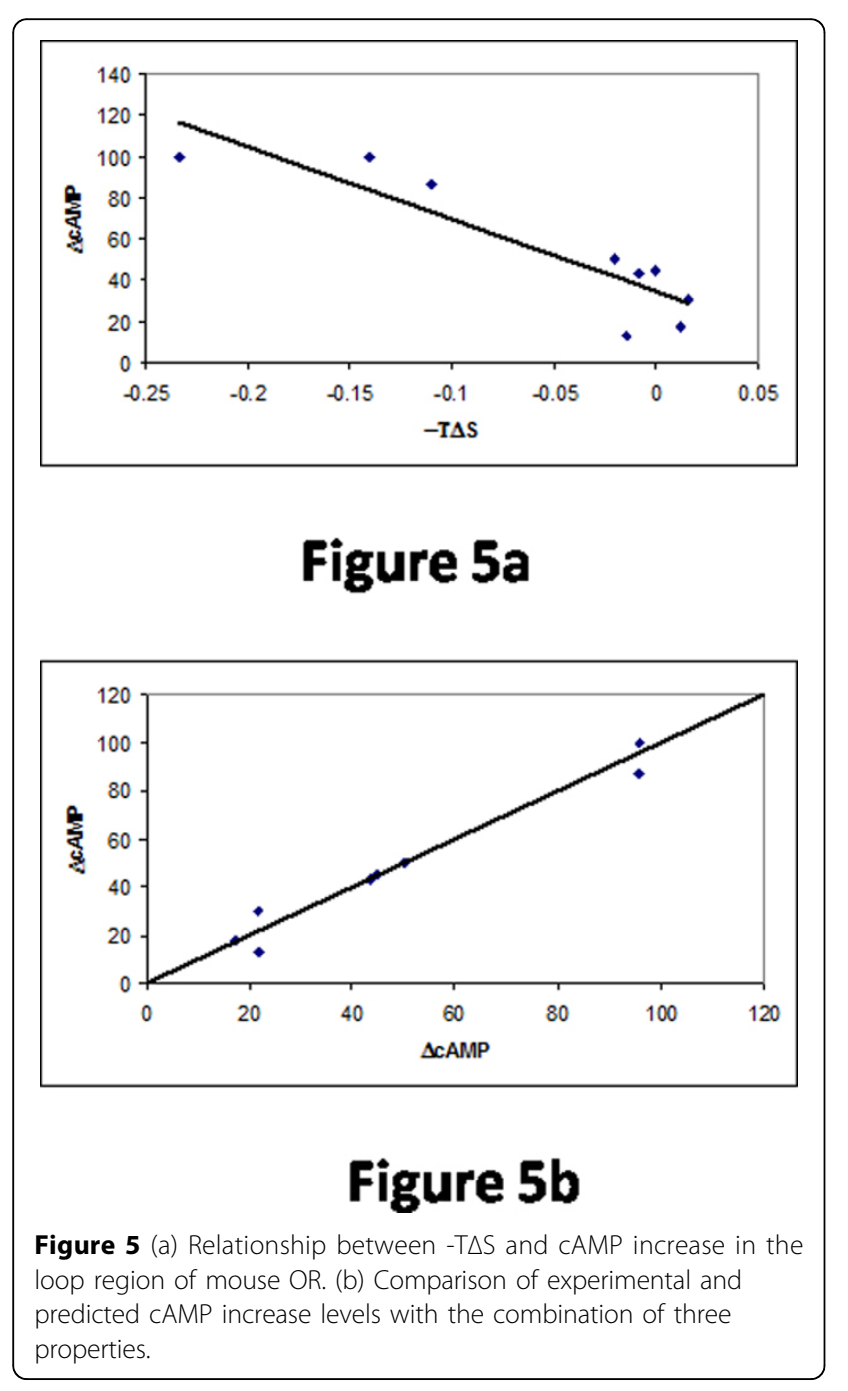

Relationship between amino acid properties and change in EC50/cAMP increase/ $\mathrm{Ca}^{2+}$ increase upon mutation: mouse OR

Katada et al. [21] measured the EC50 values for the mutants at various positions in the transmembrane helices of mouse OR. Figure 4 shows a model for mouse OR and the information about mutated residues.

We have computed the difference in amino acid properties and related with difference in EC50 values. We observed a maximum correlation of just 0.38 and the combination of five properties raised the correlation only up to 0.56 . We have included the information on neighboring residues, which increased the correlation up to 0.76 . We then tried to utilize the structural information of mouse OR using Eqn. 5. The combination of mutants, neighboring residues and surrounding residues enhanced the correlation up to 0.81 .

\section{cAMP level increase}

Kato et al. [2] measured the increase in cAMP level and $\mathrm{Ca}^{2+}$ for 24 mutants, which are located in transmembrane

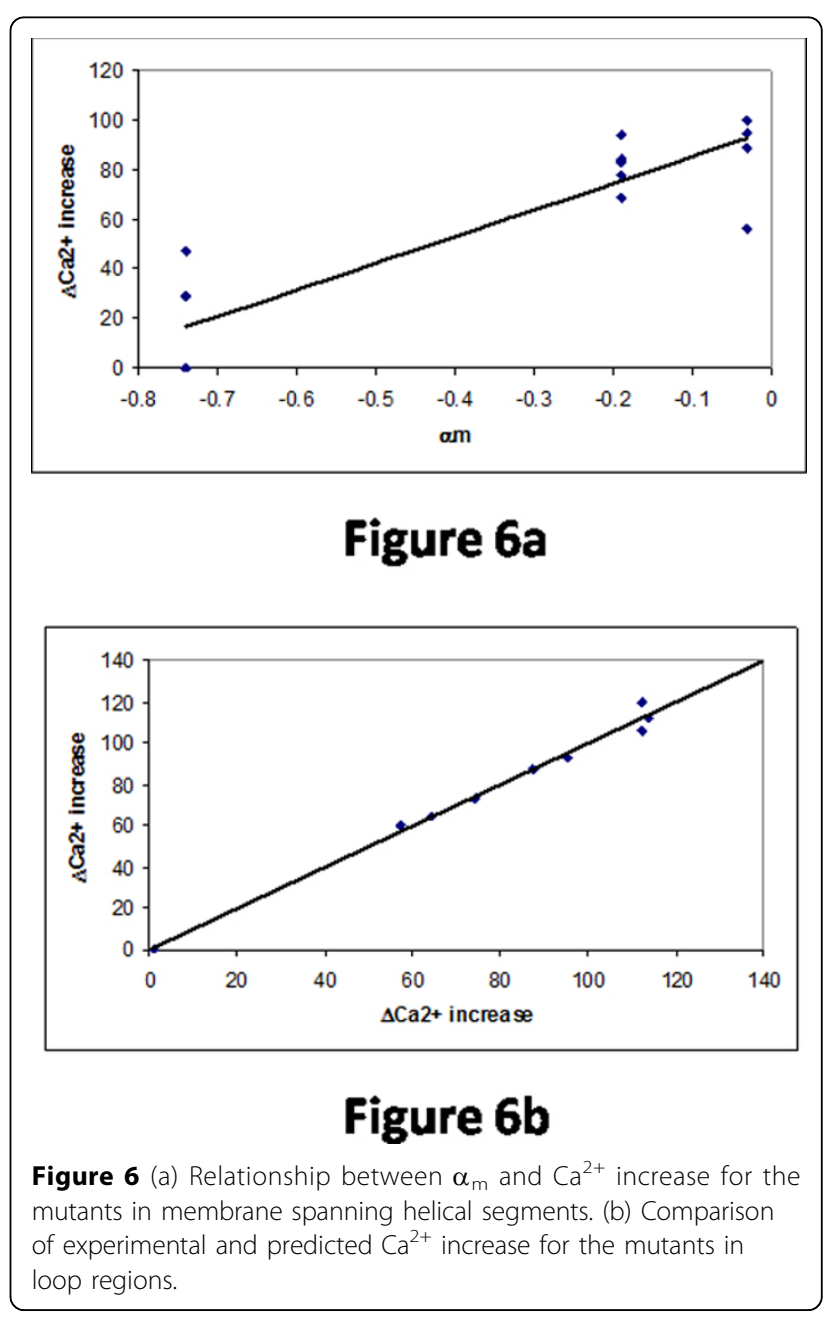


helical and loop regions. The analysis with overall data did not show high correlation and hence we classified the mutants based on their locations. Interestingly, the classification improved the correlation for the mutants both in transmembrane and loop regions.

We obtained the correlation of 0.86 with the combination of three properties for the mutants in membrane regions. In the loop region, a single property, $-\mathrm{T} \Delta \mathrm{S}$ showed a correlation of -0.89 using the information of two neighboring residues on both sides of the mutants (Figure 5a). The combination of three properties raised the correlation up to 0.98 . Figure $5 \mathrm{~b}$ shows the relationship between the experimental and predicted cAMP level increase.

We have analyzed the variation of $\mathrm{Ca}^{2+}$ increase upon mutations and we observed that the classification of mutants based on their location is necessary for understanding the properties influencing $\mathrm{Ca}^{2+}$ increase. The results obtained for transmembrane helical and loop regions are shown in Figures $6 \mathrm{a}$ and $6 \mathrm{~b}$, respectively. We obtained a maximum single correlation of 0.90 with the propensity of residues in the middle of $\alpha$-helix for the mutants in transmembrane helices (Figure 6a). The relationship between experimental and predicted $\mathrm{Ca}^{2+}$ increase for the mutants in the loop regions using a combination of three properties is shown in Figure 6b. The correlation coefficient is 0.995 .

\section{Relationship between amino acid properties and change in EC50 upon mutation: human OR}

Schmiedeberg et al. (2007) measured the EC50 values for seven mutants in human OR. We found that bulkiness has a correlation of 0.71 and the information on three neighboring residues increased the correlation up to 0.92 (Figures $7 \mathrm{a}$ and $7 \mathrm{~b}$ ). This analysis reveals the importance of neighboring residues for determining the change in EC50. Further, the combination of just two properties increased the correlation up to 0.999 and Figure $7 \mathrm{c}$ showed the relationship between experimental and predicted $\triangle \mathrm{EC} 50$.

\section{Discrimination of mutants that enhanced/decreased EC50: mouse OR}

We have collected a set of 28 data in mouse OR in which 15 of them increased EC50 upon mutation and 13 mutants decreased the EC50 values. We made an attempt to discriminate these mutants using the information on wild type residue, mutant residue and the properties of neighboring residues. We have utilized

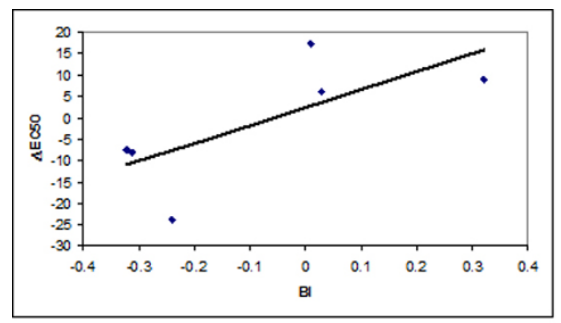

Figure 7a

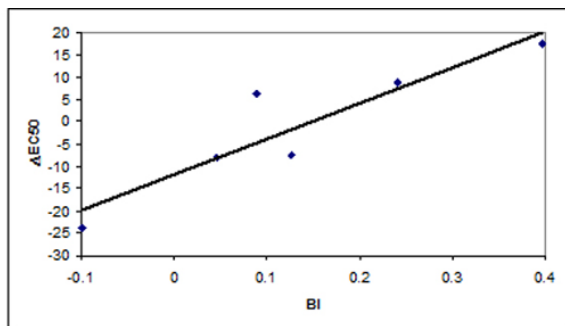

Figure 7b

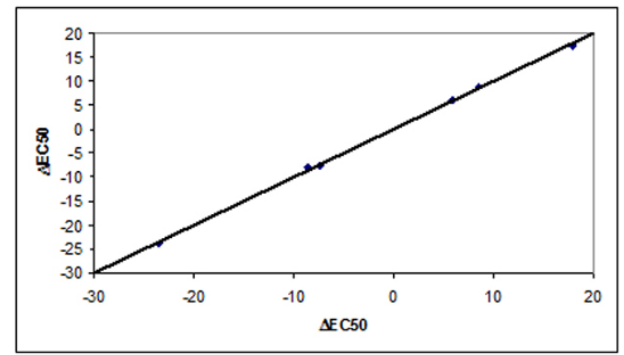

Figure 7c

Figure 7 Relationship between bulkiness and $\triangle E C 50$ in human OR; (a) Mutant information and (b) Three neighboring residues (c) Comparison of experimental and predicted $\triangle E C 50$. 
several machine learning techniques for discrimination. Our method showed an accuracy of $92.9 \%$ for self-consistency test and the sensitivity and specificity are $93.3 \%$ and 92.3\%, respectively. The assessment using jack-knife test showed an accuracy of $78.6 \%$ and the sensitivity and specificity are $80.0 \%$ and $76.9 \%$, respectively. This method can be used to identify the mutants with increased/decreased EC50.

\section{Conclusions}

We have constructed different datasets for mouse, goldfish and human ORs and various experimental data such as EC50, odorant response, $\mathrm{Ca}^{2+}$ increase etc. The experimental data have been systematically analyzed with physical, chemical, energetic and conformational properties of amino acid residues and important properties have been listed out. We found that the information on neighboring and surrounding residues, namely the inclusion of motifs, is important to understand the function. Further, we have combined the amino acid properties using multiple regression analysis, which relates experimental EC50/cAMP level increase and $\mathrm{Ca}^{2+}$ increase very well. We have utilized machine learning techniques for discriminating the mutants that enhance/reduce IC50 upon mutation and we obtained an accuracy of $93 \%$ and $79 \%$, respectively, for self-consistency and jack-knife tests. The results obtained in the present work would help to understand the importance of amino acid properties to the functions of ORs and to identify the mutants with enhanced EC50 values.

\section{Additional material}

Additional file 1: Solvent accessibility of amino acids under study.

\begin{abstract}
Acknowledgements
This research was partially supported by Indian Institute of Technology Madras research grant (BIO/10-11/540/NFSC/MICH), Indo-Japan Grant of Department of Biotechnology, India and National Institute of Advanced Industrial Science and Technology, Japan. We would like to thank IIT Madras, Computational Biology Research Center and NCBS (TIFR) for infrastructural facilities.

This article has been published as part of BMC Bioinformatics Volume 13 Supplement 7, 2012: Advanced intelligent computing theories and their applications in bioinformatics. Proceedings of the 2011 International Conference on Intelligent Computing (ICIC 2011). The full contents of the supplement are available online at http://www.biomedcentral.com/ bmcbioinformatics/supplements/13/S7.
\end{abstract}

\section{Author details \\ 'Department of Biotechnology, Indian Institute of Technology Madras, Chennai 600 036, Tamilnadu, India. ${ }^{2}$ National Center for Biological Sciences, Bangalore, India. ${ }^{3}$ Computational Biology Research Center, National Institute of Advanced Industrial Science and Technology, 2-4-7 Aomi, Koto-ku, Tokyo 135-0064, Japan.}

\section{Authors' contributions}

MMG, RS and KF conceived the project. MMG carried out the computations and wrote the manuscript. $\mathrm{KH}$ modeled the structures of ORs and analyzed them. MMG, KH, RS and FK contributed in discussions. KH and RS wrote the sections about modeling. All authors read and finalized the manuscript.

\section{Competing interests}

The authors declare that they have no competing interests.

Published: 8 May 2012

\section{References}

1. Zhao H, Ivic L, Otaki JM, Hashimoto M, Mikoshiba K, Firestein S: Functional expression of a mammalian odorant receptor. Science 1998, 279:237-242.

2. Kato A, Katada S, Touhara K: Amino acids involved in conformational dynamics and $\mathrm{G}$ protein coupling of an odorant receptor: targeting gain-of-function mutation. J Neurochem 2008, 107:1261-1270.

3. Buck LB: Information coding in the vertebrate olfactory system. Annu Rev Neurosci 1996, 19:517-544.

4. Mombaerts P: Genes and ligands for odorant, vomeronasal and taste receptors. Nat Rev Neurosci 2004, 5:263-278.

5. Leal WS: Behavioural neurobiology: The treacherous scent of a human. Nature 2010, 464:37-38

6. Carey AF, Wang G, Su CY, Zwiebel LJ, Carlson JR: Odorant reception in the malaria mosquito Anopheles gambiae. Nature 2010, 464:66-71.

7. Jaén C, Ozdener MH, Reisert J: Mechanisms of chloride uptake in frog olfactory receptor neurons. J Comp Physiol A Neuroethol Sens Neural Behav Physiol 2011, 197:339-349.

8. Hall RA: Autonomic modulation of olfactory signaling. Sci Signal 2011, 4: pe1.

9. Abuin L, Bargeton B, Ulbrich MH, Isacoff EY, Kellenberger S, Benton R: Functional architecture of olfactory ionotropic glutamate receptors. Neuron 2011, 69:44-60.

10. Ben-Chaim Y, Cheng MM, Yau KW: Unitary response of mouse olfactory receptor neurons. Proc Natl Acad Sci USA 2011, 108:822-827.

11. Baud O, Etter S, Spreafico M, Bordoli L, Schwede T, Vogel H, Pick H: The mouse eugenol odorant receptor: structural and functional plasticity of a broadly tuned odorant binding pocket. Biochemistry 2011, 50:843-853.

12. Ezkurdia L, Bartoli L, Fariselli P, Casadio R, Valencia A, Tress ML: Progress and challenges in predicting protein-protein interaction sites. Brief Bioinform 2009, 10:233-246.

13. Yanay O, Rost B: Protein-protein interaction hotspots carved into sequences. PLoS Comput Biol 2007, 3:e119.

14. You ZH, Lei YK, Huang DS, Zhou X: Using manifold embedding for assessing and predicting protein interactions from high-throughput experimental data. Bioinformatics 2010, 26(21):2744-2751.

15. Xia JF, Zhao XM, Song J, Huang DS: APIS: accurate prediction of hot spots in protein interfaces by combining protrusion index with solvent accessibility. BMC Bioinformatics 2010, 11:174.

16. Xia JF, Zhao XM, Huang DS: Predicting protein-protein interactions from protein sequences using meta predictor. Amino Acids 2010, 39:1595-1599.

17. Gromiha MM, Yokota K, Fukui K: Energy based approach for understanding the recognition mechanism in protein-protein complexes. Mol Biosyst 2009, 5(12):1779-1786.

18. Gromiha MM, Yabuki Y, Suresh MX, Thangakani AM, Suwa M, Fukui K: TMFunction: database for functional residues in membrane proteins. Nucleic Acids Res 2009, 37:D201-D204.

19. Kuang D, Yao Y, Wang M, Pattabiraman N, Kotra LP, Hampson DR Molecular similarities in the ligand binding pockets of an odorant receptor and the metabotropic glutamate receptors. J Biol Chem 2003, 278:42551-42559.

20. Luu P, Acher F, Bertrand HO, Fan J, Ngai J: Molecular determinants of ligand selectivity in a vertebrate odorant receptor. J Neurosci 2004 , 24:10128-10137.

21. Katada S, Hirokawa T, Oka Y, Suwa M, Touhara K: Structural basis for a broad but selective ligand spectrum of a mouse olfactory receptor: mapping the odorant-binding site. J Neurosci 2005, 25:1806-1815.

22. Schmiedeberg K, Shirokova E, Weber HP, Schilling B, Meyerhof W, Krautwurst D: Structural determinants of odorant recognition by the human olfactory receptors OR1A1 and OR1A2. J Struct Biol 2007, 159:400-412.

23. Palczewski K, Kumasaka T, Hori T, Behnke CA, Motoshima H, Fox BA, Le Trong I, Teller DC, Okada T, Stenkamp RE, Yamamoto M, Miyano M: Crystal 
structure of rhodopsin: a G protein-coupled receptor. Science 2000, 289:739-745.

24. Man O, Gilad Y, Lancet D: Prediction of the odorant binding site of olfactory receptor proteins by human-mouse comparisons. Protein $\mathrm{Sci}$ 2004, 13:240-254.

25. Olender $\mathrm{T}$, Feldmesser $\mathrm{E}$, Atarot $\mathrm{T}$, Eisenstein M, Lancet $\mathrm{D}$ : The olfactory receptor universe - from whole genome analysis to structure and evolution. Genet Mol Res 2004, 3:545-553.

26. Tomii K, Kanehisa M: Analysis of amino acid indices and mutation matrices for sequence comparison and structure prediction of proteins. Protein Eng 1996, 9:27-36

27. Gromiha MM, Oobatake M, Sarai A: Important amino acid properties for enhanced thermostability from mesophilic to thermophilic proteins. Biophys Chem 1999, 82:51-67.

28. Gromiha MM, Oobatake M, Kono $H$, Uedaira H, Sarai A: Importance of surrounding residues for protein stability of partially buried mutations. J Biomol Struct Dyn 2000, 18:281-295.

29. Gromiha MM, Oobatake $M$, Kono $H$, Uedaira $H$, Sarai A: Role of structural and sequence information in the prediction of protein stability changes: comparison between buried and partially buried mutations. Protein Eng 1999, 12:549-555.

30. Gromiha MM, Selvaraj S: Important amino acid properties for determining the transition state structures of two-state protein mutants. FEBS Lett 2002, 526:129-134

31. Huang LT, Gromiha MM: Reliable prediction of protein thermostability change upon double mutation from amino acid sequence. Bioinformatics 2009, 25:2181-2187.

32. Huang LT, Gromiha MM: First insight into the prediction of protein folding rate change upon point mutation. Bioinformatics 2010 26:2121-2127.

33. Ou YY, Chen SA, Gromiha MM: Classification of transporters using efficient radial basis function networks with position-specific scoring matrices and biochemical properties. Proteins 2010, 78:1789-1797.

34. Tusndy GE, Simon I: The HMMTOP transmembrane topology prediction server. Bioinformatics 2001, 17:849-850.

35. Pirovano W, Feenstra KA, Heringa J: PRALINETM: a strategy for improved multiple alignment of transmembrane proteins. Bioinformatics 2008, 24(2):492-497.

36. Waterhouse AM, Procter JB, Martin DMA, Clamp M, Barton GJ: Jalview version 2-a multiple sequence alignment editor and analysis workbench. Bioinformatics 2009, 25:1189-1191, doi: 10.1093/bioinformatics/ btp033.

37. Sali A, Blundell TL: Comparative protein modelling by satisfaction of spatial restraints. J Mol Biol 1993, 234:779-815.

38. Laskowski RA, MacArthur MW, Moss DS, Thornton JM: PROCHECK: a program to check the stereochemical quality of protein structures. J Appl Crystallogr 1993, 26:283-291.

39. Gromiha MM, Selvaraj S: Inter-residue interactions in protein folding and stability. Prog Biophys Mol Biol 2004, 86:235-277.

40. Gromiha MM: Influence of long-range contacts and surrounding residues on the transition state structures of proteins. Anal Biochem 2011, 408:32-36.

41. Grewal PS: Numerical Methods of Statistical Analysis New Delhi: Sterling Publ; 1987.

42. Witten IH, Frank E: Data Mining: Practical Machine Learning Tools and Techniques. 2 edition. San Francisco: Morgan Kaufmann; 2005.

43. Gromiha MM, Suwa M: Discrimination of outer membrane proteins using machine learning algorithms. Proteins 2006, 63:1031-1037.

44. Gromiha MM, Yabuki Y: Functional discrimination of membrane proteins using machine learning techniques. BMC Bioinformatics 2008, 9:135.

45. Gromiha MM, Suwa M: Influence of amino acid properties for discriminating outer membrane proteins at better accuracy. Biochim Biophys Acta 2006, 1764:1493-1497.

46. Gromiha MM, Sowdhamini R, Fukui K: Structure-function relationship in olfactory receptors. Lect Notes Bioinf 2011, 6840:618-623.

47. Lee B, Richards FM: The interpretation of protein structures: estimation of static accessibility. J Mol Biol 1971, 55(3):379-400.

48. Overington J, Johnson MS, Sali A, Blundell TL: Tertiary structural constraints on protein evolutionary diversity: templates, key residues and structure prediction. Proc Biol Sci 1990, 241:132-145.
49. Mizuguchi K, Deane CM, Blundell TL, Johnson MS, Overington JP: JOY: protein sequence-structure representation and analysis. Bioinformatics $1998,14: 617-623$.

doi:10.1186/1471-2105-13-S7-S1

Cite this article as: Gromiha et al:: Relationship between amino acid properties and functional parameters in olfactory receptors and discrimination of mutants with enhanced specificity. BMC Bioinformatics 2012 13(Suppl 7):S1.

\section{Submit your next manuscript to BioMed Central and take full advantage of:}

- Convenient online submission

- Thorough peer review

- No space constraints or color figure charges

- Immediate publication on acceptance

- Inclusion in PubMed, CAS, Scopus and Google Scholar

- Research which is freely available for redistribution 